\title{
Imaging of cardiac sympathetic activity in heart failure: Not out of the woods yet
}

\author{
Catherine Gebhard, $M^{a}$ \\ a Department of Nuclear Medicine, University Hospital Zurich, Zurich, Switzerland
}

Received Jan 4, 2017; accepted Jan 4, 2017

doi:10.1007/s12350-017-0779-1

\section{See related article, pp. 1164-1171}

Currently, 5.7 million people are living with heart failure (HF) in the U.S., and given the aging of the population, the prevalence of HF continues to grow. ${ }^{1}$ In addition, $\mathrm{HF}$ is the leading cause of hospitalizations in people over the age of 65 , thereby posing an increasing problem for global healthcare systems. ${ }^{2}$ In view of this burden, risk-based, personalized therapeutic strategies and a cost-effective management for patients with $\mathrm{HF}$ are needed. However, despite notable improvements in medical therapy, outcomes for $\mathrm{HF}$ patients are still unacceptable and precise risk stratification in $\mathrm{HF}$ remains challenging. ${ }^{2}$ In fact, various prognostic markers of mortality and morbidity of HF have been identified; however, their clinical applicability is limited. Similarly, tools contributing to the appropriate selection of therapeutic strategies or monitoring their efficacy are lacking. There is accumulating evidence that imaging of cardiac sympathetic activity by ${ }^{123} \mathrm{I}-$ metaiodobenzylguanidine ( $m \mathrm{IBG})$, a sympathetic neurotransmitter radionuclide analog (Figure 1), may help to direct therapy and clinical decision-making in $\mathrm{HF}$ patients. Indeed, given that dysfunction of the sympathetic nervous system is one of the key features of worsening systolic HF, pharmacological and non-pharmacological targeting of sympathetic dysfunction has emerged as a promising treatment strategy in $\mathrm{HF}^{2}$

This editorial refers to: "Impact of an exercise-training programme on cardiac neuronal function in $\mathrm{HF}$ patients on optimal medical therapy - A randomized iodine-123 MIBG scintigraphy study" by Valborgland et al.

Reprint requests: Catherine Gebhard, MD, Department of Nuclear Medicine, University Hospital Zurich, Raemistrasse 100, 8091, Zurich, Switzerland; catherine.gebhard@usz.ch

J Nucl Cardiol 2018;25:1172-7.

1071-3581/\$34.00

Copyright (C) 2017 American Society of Nuclear Cardiology.
Accordingly, ${ }^{123}$ I- $m$ IBG imaging has been shown to have an incremental prognostic value in predicting disease progression, arrhythmic events, and cardiac death in HF patients beyond that provided by traditional functional and neurohormonal markers. ${ }^{3}$ However, despite its prognostic importance and its FDA approval for cardiac applications in 2013, the exact role of cardiac neuronal imaging in diagnosis and management of HF is still under debate, and therefore, ${ }^{123} \mathrm{I}-m \mathrm{IBG}$ scintigraphy is currently mostly applied as a research tool.

In this issue of the Journal of Nuclear Cardiology, Valborgland et al. evaluate the effect of aerobic exercise training on cardiac sympathetic activity in twenty-three HF patients receiving optimal medical therapy including $\beta$-blockers. All patients were submitted to planar ${ }^{123} \mathrm{I}-m \mathrm{IBG}$ scintigraphy at baseline and after 12 weeks of exercise training. The authors found that different types of exercise training did not significantly change cardiac neuronal uptake as quantified by Heart-Mediastinum Ratio (HMR) in these patients. Given the strong and positive association between regular aerobic exercise and improved outcomes $^{4,5}$ as well as the restoration of resting cardiac sympathetic activity following exercise in $\mathrm{HF}^{6}{ }^{6}$ these results are surprising. Importantly, one of the major advances in HF treatment during the past decade was the implementation of $\beta$-adrenergic-blocking agents in therapeutic strategies, which has substantially improved clinical outcomes in these patients. ${ }^{7,8}$ This beneficial effect seems to be driven by an improvement of cardiac sympathetic dysfunction. ${ }^{9}$ Valborgland et al. now provide evidence against a synergistic effect of exercise training and contemporary pharmacotherapy on sympathetic activation in $\mathrm{HF}$ by demonstrating that the hemodynamic and metabolic adaptations induced by 12 weeks of exercise training do not further improve cardiac sympathetic dysfunction in HF patients already on $\beta$-blocking therapy. As stated by the authors, these data contrast with an earlier report demonstrating a favorable effect 


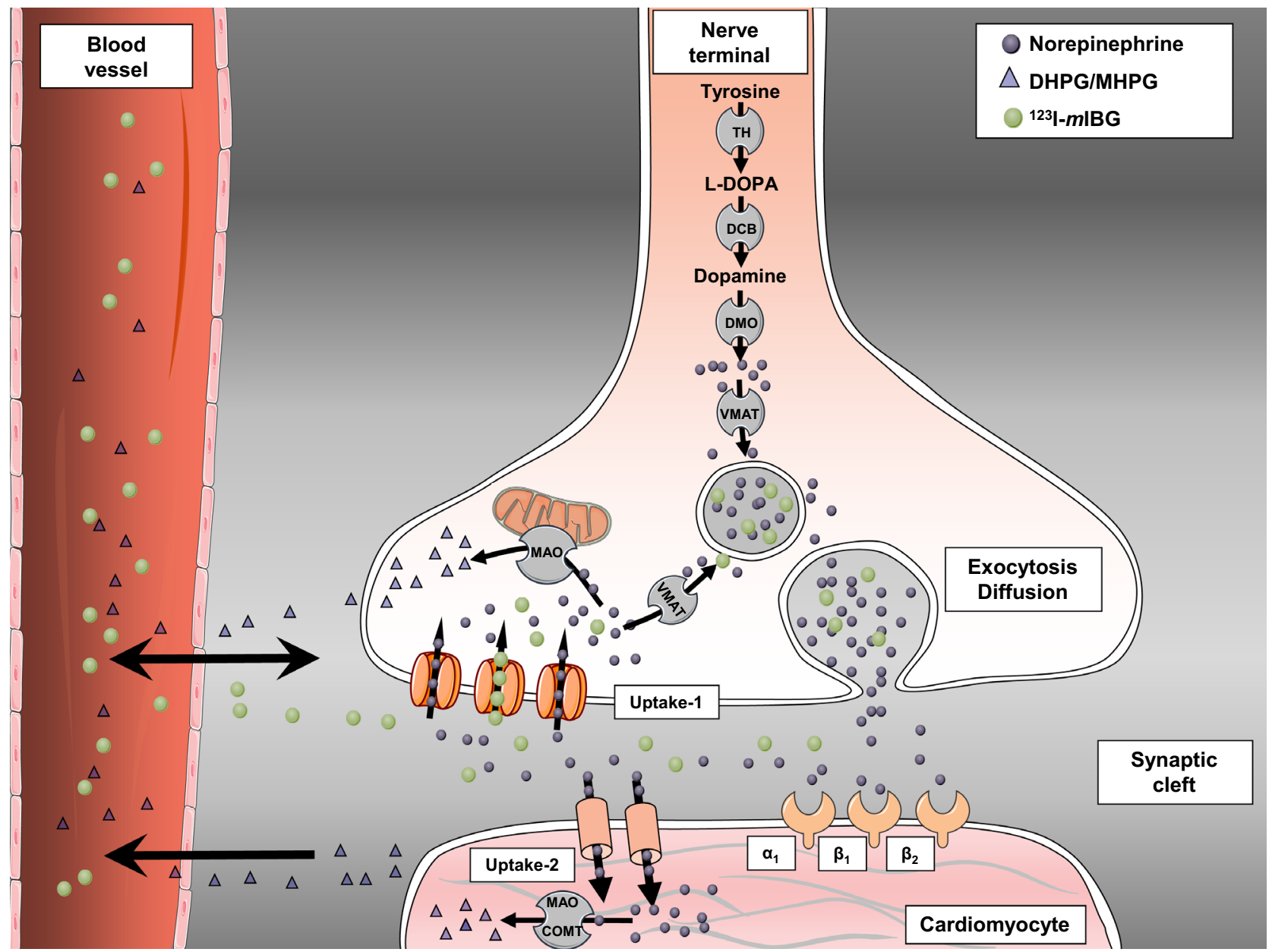

Figure 1. Schematic description of norepinephrine and ${ }^{123}$ I-Metaiodobenzylguanidine ( $\left.m \mathrm{IBG}\right)$ kinetics in sympathetic nerve ending. Norepinephrine is synthesized in presynaptic neurons from tyrosine by dopamine $\beta$-monooxygenase (DMO) via L-DOPA (tyrosine hydroxylase, TH) and dopamine (DOPA decarboxylase, DCB), then transported into presynaptic neuronal terminal vesicles via vesicular monoamine transporter (VMAT), and finally released into the synaptic cleft via exocytosis/diffusion. Only a small percentage of norepinephrine binds to postsynaptic $\alpha 1-, \beta 1-$, and $\beta 2$-adrenoceptors for downstream effects, while the majority of norepinephrine is reabsorbed into the presynaptic neuron via uptake- 1 transporter. Most of the norepinephrine is stored again in vesicles while $5 \%$ to $10 \%$ of norepinephrine is metabolized to dihydroxyphenylglycol (DHPG) by monoamine oxidase (MAO) and released into the circulation. In addition, norepinephrine can be absorbed in non-neuronal tissue (e.g., cardiomyocytes) via the uptake- 2 mechanism. In these tissues, norepinephrine is metabolized to DHPG (via MAO) and then to 3-methoxy-4hydroxyphenylglycol (MHPG) by catechol-O-methyl transferase (COMT). ${ }^{123} \mathrm{I}-m \mathrm{IBG}$ is chemically modified from guanethidine which is an analog of norepinephrine. ${ }^{123} \mathrm{I}-\mathrm{mIBG}$ uses the same transporters (mainly uptake-1) and storage mechanisms like norepinephrine. Unlike norepinephrine, ${ }^{123} \mathrm{I}-m \mathrm{IBG}$ is not metabolized by MAO and COMT and does not bind to postsynaptic adrenergic receptors.

of exercise training on cardiac neuronal dysfunction in HF patients not on $\beta$-blocking therapy. ${ }^{6}$ In addition, it has been shown that exercise training reduces muscle sympathetic nerve activity (MSNA) even in HF patients receiving $\beta$-blocker treatment. ${ }^{10}$ Of note, however, in contrast to quantification of sympathetic activity in individual organs, MSNA does not provide information on regional sympathetic outflow. Given that the magnitude of sympathetic activation in $\mathrm{HF}$ is target-organ specific, results may substantially differ. Importantly, while no changes in HMR or tracer washout rate (WR) were detected, Valborgland et al. observed a significant improvement in 6-minute walk test, peak oxygen uptake, and maximal work-load following exercise, indicating that the exercise regimen applied in their study further enhanced functional 
capacity in these patients. These intriguing results leave us with the question why there is a discrepancy between changes in functional capacity and cardiac innervation imaging in the response to exercise training? And might pathophysiological or technical factors account for these findings?

The benefits of exercise training in chronic HF have been extensively studied and comprise, besides reducing sympathetic outflow, an increase in renal blood flow as well as improved endothelial and muscle function, reduction of reactive oxygen species (ROS), circulating cytokines, angiotensin II and aldosterone levels, enhanced baro- and chemoreflex sensitivity, and an increase in vagal tone (Figure 2). ${ }^{11,12}$ While there is clear evidence that exercise-induced adaptions in $\mathrm{HF}$ are strongly interrelated, the relative contributions of these variables to improved outcomes in HF patients are not well understood. Indeed, a downregulation of sympathetic neuronal activity via central angiotensin II receptors and central antioxidant mechanisms has been shown, and studies have demonstrated an improvement of cardiac sympathetic dysfunction by angiotensin-converting enzyme (ACE) inhibitors and aldosterone, which do not directly affect myocardial adrenergic functions (Figure 2). ${ }^{13-16}$ However, a cause-effect relationship between these variables is yet to be established. Following this line of reasoning, contemporary pharmacotherapy including inhibitors of the renin-angiotensin-aldosterone system (RAAS) and $\beta$-blockers may substantially change the relative impact of different pathways and organ systems on exercise-induced adaptions in HF and may, as demonstrated by Valborgland et al., result in a greater exercise-induced effect on peripheral circulatory systems as compared to cardiac neuronal activation. Thus, although these data should be approached cautiously because of the small sample size, the most important contribution of Valborgland et al. is the demonstration that restoration of autonomic function following exercise training might substantially differ in HF patients with and without maximal pharmacotherapy. In this regard, it must be emphasized that the best response variable for the assessment of the efficacy of a treatment in HF patients is still under debate. ${ }^{17}$ Thus, knowledge about the exact organ system and specific pathways mediating the benefits of a therapeutic intervention in $\mathrm{HF}$ will lay the foundation for precision medicine and improved clinical endpoints in HF.

The merit of the study by Valborgland et al. also lies in the fact that current limitations of ${ }^{123} \mathrm{I}-m \mathrm{IBG}$ imaging have been depicted. In fact, only few studies have assessed whether ${ }^{123} \mathrm{I}-m \mathrm{IBG}$ imaging might help to decide who might benefit from a particular therapy. ${ }^{18,19}$ In addition, there are no clinical data using cardiac MIBG imaging for drug intervention in $\mathrm{HF}$ patients.
Thus, the effect of ${ }^{123}$ I- $m$ IBG scintigraphy on patient's management and selection of therapy is currently unknown. Given the high benefit to risk/cost ratio of conventional medical therapies, ${ }^{123} \mathrm{I}-m \mathrm{IBG}$ imaging is currently unlikely to preclude their use. The present study by Valborgland et al. further supports this notion by indicating that the assessment of functional parameters might be a more sensitive test for a treatment response than cardiac neuronal imaging. This leads us to the ultimate question whether there is a role for ${ }^{123} \mathrm{I}-\mathrm{mIBG}$ imaging in selecting an appropriate therapeutic strategy in HF patients. Noteworthy, despite improved therapeutic options in $\mathrm{HF}$, the risk reduction rate of current HF drugs is still limited and drug intolerance and adverse effects are common. In addition, non-responders to therapeutic interventions in HF have been identified in previous studies. ${ }^{20}$ Finally, wide variations in HF disease phenotypes and the abundance of comorbidities in HF patients undoubtedly necessitate better-informed decision-making and a personalized disease management. Thus, given its ability to trace alterations of cardiac sympathetic nerve function caused by drug interventions, ${ }^{18}{ }^{123} \mathrm{I}-m \mathrm{IBG}$ imaging might have a clinical value in optimizing therapy in specific subpopulations such as patients not responding to routine therapeutic strategies or in patients in whom intolerable side effects prohibit adequate pharmacological dosing. In this regard, cardiac neuroimaging has been attributed a potential role in selecting patients likely to benefit from device therapy, given its value as a negative predictor for life-threatening arrhythmias. ${ }^{3}$ However, optimal cutoff values to identify these patients are lacking; therefore large-scale, interventional studies are needed to strengthen these findings.

Valborgland et al. conclude the discussion of their study by wondering whether a significant effect of exercise training on cardiac sympathetic activity would have been detected in a more deconditioned HF population such as patients classified in New York Heart Association (NYHA) class III or IV. Another reason for their neutral findings might be that the level of exercise applied in the present study was insufficient to induce significant changes in cardiac sympathetic activation. In fact, as acknowledged by the authors, previous studies have applied longer treatment durations when assessing the effect of exercise training on HF outcomes. ${ }^{6}$ Alternatively, pertaining to the small sample size of the study, individual variations might have had a significant confounding influence on cardiac sympathoexcitatory measures. Indeed, comorbidities such as obesity, sleep apnea, hypertension, myocardial ischemia, and diabetes, each of which have been shown to elevate the set point for sympathetic outflow, are common in HF patients. ${ }^{21,22}$ Thus, sympathetic tone differs widely among patients 


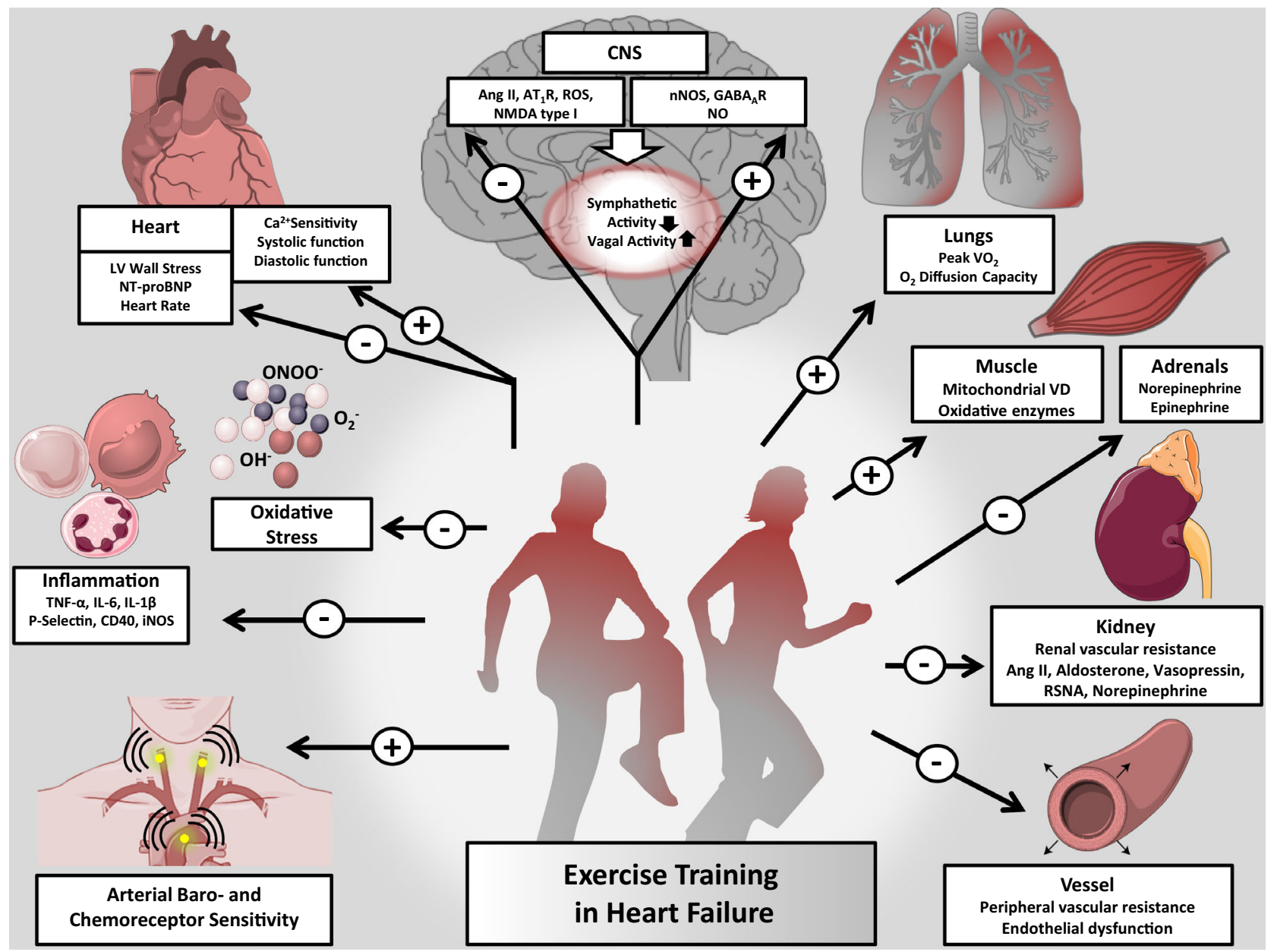

Figure 2. Effects of exercise training in systolic heart failure. N-terminal pro b-type natriuretic peptide. $C N S$ central nervous system, $L V$ left ventricular, $T N F-\alpha$ tumor necrosis factor- $\alpha, I L$ interleukin, $i N O S$ inducible nitric oxide synthase, $n N O S$ neuronal nitric oxide synthase, $N T$-proBNP $\mathrm{N}$-terminal pro b-type natriuretic peptide, $N O$ nitric oxide, $G A B A_{A} R \gamma$-aminobutyric acid A receptor, Ang II angiotensin II, MitoVD mitochondrial volume density, RSNA renal sympathetic nerve activity.

with $\mathrm{HF}$ and large inter-individual variations of myocardial ${ }^{123} \mathrm{I}-m \mathrm{IBG}$ uptake have also been reported in normal subjects. ${ }^{23}$ These variations seem to be the result of both, methodological and (patho)physiological factors and need to be taken into account when interpreting ${ }^{123} \mathrm{I}$ - $m$ IBG images. ${ }^{24}$ However, technical refinements in ${ }^{123} \mathrm{I}-m \mathrm{IBG}$ imaging such as iterative reconstruction, volumetric analysis techniques as well as automated quantitation have been introduced in recent years. In addition, the need to standardize myocardial ${ }^{123} \mathrm{I}-m \mathrm{IBG}$ scintigraphy has recently been emphasized. ${ }^{25}$ These current developments will certainly help to overcome the above limitations and to pave the way for a clinical implementation of ${ }^{123} \mathrm{I}-m \mathrm{IBG}$ imaging.

Pertaining to a wider clinical use of cardiac ${ }^{123}$ I- $m$ IBG imaging, it will also be indispensable to consider the influence of sex and age on cardiac sympathetic activity in HF. The study of Valborgland et al. could not address this important issue, given the low number of females $(n=1)$ included in the study. Notably, sexual dimorphism in autonomic nervous control of the cardiovascular system has previously been demonstrated and has gained increasing attention in the context of Takotsubo cardiomyopathy. ${ }^{26}$ The fact that women with ischaemic HF exhibit increased cardiacspecific sympathetic activation and have worse outcomes than men, once affected, implies that this sexual dimorphism in cardiac autonomic physiology is pathogenetic. $^{27}$ Of note, sex-specific differences in the predictive power of peak $\mathrm{VO}_{2}$ measures in $\mathrm{HF}$ have also been described and lower cut-off values for women with HF have been suggested. ${ }^{28,29}$ In addition, several peculiarities with regard to sympathetic activation in older people need to be considered, such as a higher 
sympathetic nervous activity at baseline and in response to stress as compared to younger subjects. ${ }^{30}$ Thus, before ${ }^{123}$ I- $m$ IBG imaging can become a valuable addition to clinical practice in the treatment of $\mathrm{HF}$, sufficiently powered studies are necessary to determine whether gender- and age-specific cutoff values are needed.

In summary, Valborgland et al. take another step forward in providing novel and important information indicating that the impact of a therapeutic intervention on autonomic dysfunction in HF might be influenced by concomitant medication. While their results require confirmation in additional studies where the abovementioned limitations should be addressed, they also point out that understanding the multifactorial nature of cardiac autonomic modulation will continue to provide pathophysiologic and therapeutic insights for HF management. Larger clinical trials will be required to test whether ${ }^{123}$ I- $m$ IBG imaging might indeed be of value in better selecting patients who may benefit from additional treatment. Such an individualized treatment approach will become ever more important in the upcoming years, given the aging of the population and the increasing burden of HF. Therefore, specific subgroups, such as elderly and females, should also be investigated in more detail. As a priority for future research, standardization of myocardial ${ }^{123} \mathrm{I}-\mathrm{mIBG}$ scintigraphy should be achieved in order to improve its clinical utility. Thus, for the time being, we should withhold the final word on the clinical utility of cardiac ${ }^{123} \mathrm{I}-m \mathrm{IBG}$ imaging and await further data linking treatment responses with ${ }^{123} \mathrm{I}-m \mathrm{IBG}$ images and, ideally, with future cardiovascular events.

\section{Funding}

This work was supported by grants from the Swiss National Science Foundation (SNSF), the Olga Mayenfisch Foundation, Switzerland, and the Swissheart Foundation.

\section{Disclosure}

None.

\section{References}

1. Mozaffarian D, Benjamin EJ, Go AS, Arnett DK, Blaha MJ, Cushman M, et al. Heart disease and stroke statistics-2016 update: a report from the American Heart Association. Circulation. 2016;133:e38-360. doi:10.1161/CIR.0000000000000350.

2. Ponikowski P, Voors AA, Anker SD, Bueno H, Cleland JG, Coats AJ, et al. 2016 ESC Guidelines for the diagnosis and treatment of acute and chronic heart failure: the Task Force for the diagnosis and treatment of acute and chronic heart failure of the European Society of Cardiology (ESC) developed with the special contribution of the Heart Failure Association (HFA) of the ESC. Eur Heart J. 2016;37:2129-200.
3. Jacobson AF, Senior R, Cerqueira MD, Wong ND, Thomas GS, Lopez VA, et al. Myocardial iodine-123 meta-iodobenzylguanidine imaging and cardiac events in heart failure. Results of the prospective ADMIRE-HF (AdreView Myocardial Imaging for Risk Evaluation in Heart Failure) study. J Am Coll Cardiol. 2010;55:2212-21.

4. Belardinelli R, Georgiou D, Cianci G, Purcaro A. Randomized, controlled trial of long-term moderate exercise training in chronic heart failure: effects on functional capacity, quality of life, and clinical outcome. Circulation. 1999;99:1173-82.

5. O'Connor CM, Whellan DJ, Lee KL, Keteyian SJ, Cooper LS, Ellis SJ, et al. Efficacy and safety of exercise training in patients with chronic heart failure: HF-ACTION randomized controlled trial. JAMA. 2009;301:1439-50.

6. Agostini D, Lecluse E, Belin A, Babatasi G, Amar MH, Grollier $\mathrm{G}$, et al. Impact of exercise rehabilitation on cardiac neuronal function in heart failure: an iodine-123 metaiodobenzylguanidine scintigraphy study. Eur J Nucl Med. 1998;25:235-41.

7. Packer M, Bristow MR, Cohn JN, Colucci WS, Fowler MB, Gilbert EM, et al. The effect of carvedilol on morbidity and mortality in patients with chronic heart failure. U.S. Carvedilol Heart Failure Study Group. N Engl J Med. 1996;334:1349-55.

8. The Cardiac Insufficiency Bisoprolol Study II. (CIBIS-II): a randomised trial. Lancet. 1999;353:9-13.

9. Chizzola PR, Goncalves de Freitas HF, Marinho NV, Mansur JA, Meneghetti JC, Bocchi EA. The effect of beta-adrenergic receptor antagonism in cardiac sympathetic neuronal remodeling in patients with heart failure. Int J Cardiol. 2006;106:29-34.

10. Fraga R, Franco FG, Roveda F, de Matos LN, Braga AM, Rondon $\mathrm{MU}$, et al. Exercise training reduces sympathetic nerve activity in heart failure patients treated with carvedilol. Eur J Heart Fail. 2007;9:630-6.

11. Demopoulos L, Bijou R, Fergus I, Jones M, Strom J, LeJemtel TH. Exercise training in patients with severe congestive heart failure: enhancing peak aerobic capacity while minimizing the increase in ventricular wall stress. J Am Coll Cardiol. 1997;29:597-603.

12. Minotti JR, Johnson EC, Hudson TL, Zuroske G, Murata G, Fukushima E, et al. Skeletal muscle response to exercise training in congestive heart failure. J Clin Invest. 1990;86:751-8.

13. Somsen GA, van Vlies B, de Milliano PA, Borm JJ, van Royen EA, Endert E, et al. Increased myocardial $\left[{ }^{123} \mathrm{I}\right]$-metaiodobenzylguanidine uptake after enalapril treatment in patients with chronic heart failure. Heart. 1996;76:218-22.

14. Kasama S, Toyama T, Kumakura H, Takayama Y, Ichikawa S, Suzuki T, et al. Effect of spironolactone on cardiac sympathetic nerve activity and left ventricular remodeling in patients with dilated cardiomyopathy. J Am Coll Cardiol. 2003;41:574-81.

15. Mousa TM, Liu D, Cornish KG, Zucker IH. Exercise training enhances baroreflex sensitivity by an angiotensin II-dependent mechanism in chronic heart failure. J Appl Physiol. 1985;2008(104):616-24.

16. Gao L, Wang W, Liu D, Zucker IH. Exercise training normalizes sympathetic outflow by central antioxidant mechanisms in rabbits with pacing-induced chronic heart failure. Circulation. 2007;115:3095-102.

17. Neaton JD, Gray G, Zuckerman BD, Konstam MA. Key issues in end point selection for heart failure trials: composite end points. $\mathbf{J}$ Card Fail. 2005;11:567-75.

18. Fukuoka S, Hayashida K, Hirose Y, Shimotsu Y, Ishida Y, Kakuchi $\mathrm{H}$, et al. Use of iodine-123 metaiodobenzylguanidine myocardial imaging to predict the effectiveness of beta-blocker therapy in patients with dilated cardiomyopathy. Eur J Nucl Med. 1997;24:523-9. 
19. Suwa M, Otake Y, Moriguchi A, Ito T, Hirota Y, Kawamura K, et al. Iodine-123 metaiodobenzylguanidine myocardial scintigraphy for prediction of response to beta-blocker therapy in patients with dilated cardiomyopathy. Am Heart J. 1997;133:353-8.

20. Stoylen A, Conraads V, Halle M, Linke A, Prescott E, Ellingsen O. Controlled study of myocardial recovery after interval training in heart failure: SMARTEX-HF-rationale and design. Eur J Prev Cardiol. 2012;19:813-21.

21. Ferrier C, Esler MD, Eisenhofer G, Wallin BG, Horne M, Cox HS, et al. Increased norepinephrine spillover into the jugular veins in essential hypertension. Hypertension. 1992;19:62-9.

22. Rumantir MS, Vaz M, Jennings GL, Collier G, Kaye DM, Seals DR, et al. Neural mechanisms in human obesity-related hypertension. J Hypertens. 1999;17:1125-33.

23. Tsuchimochi S, Tamaki N, Tadamura E, Kawamoto M, Fujita T, Yonekura $\mathrm{Y}$, et al. Age and gender differences in normal myocardial adrenergic neuronal function evaluated by iodine-123MIBG imaging. J Nucl Med. 1995;36:969-74.

24. Chirumamilla A, Travin MI. Cardiac applications of ${ }^{123} \mathrm{I}-\mathrm{mIBG}$ imaging. Semin Nucl Med. 2011;41:374-87.
25. Verberne HJ. Assessment of cardiac sympathetic innervation with (123)I-mIBG SPECT comes to life: need for standardization! Eur Heart J Cardiovasc Imaging. 2016;17:391-2.

26. Christou DD, Jones PP, Jordan J, Diedrich A, Robertson D, Seals DR. Women have lower tonic autonomic support of arterial blood pressure and less effective baroreflex buffering than men. Circulation. 2005;111:494-8.

27. Mitoff PR, Gam D, Ivanov J, Al-hesayen A, Azevedo ER, Newton GE, et al. Cardiac-specific sympathetic activation in men and women with and without heart failure. Heart. 2011;97:382-7.

28. Keteyian SJ, Patel M, Kraus WE, Brawner CA, McConnell TR, Pina IL, et al. Variables measured during cardiopulmonary exercise testing as predictors of mortality in chronic systolic heart failure. J Am Coll Cardiol. 2016;67:780-9.

29. Elmariah S, Goldberg LR, Allen MT, Kao A. Effects of gender on peak oxygen consumption and the timing of cardiac transplantation. J Am Coll Cardiol. 2006;47:2237-42.

30. Esler MD, Thompson JM, Kaye DM, Turner AG, Jennings GL, Cox HS, et al. Effects of aging on the responsiveness of the human cardiac sympathetic nerves to stressors. Circulation. 1995;91:351-8. 